\title{
Cranial CT Evaluation of Linear Measurements in Children with Hydrocephalus in Sokoto, Nigeria
}

\author{
Gele IH, Saidu SA, Ma'aji SM, Danfulani M, Yunusa GH, Muhammad SB \\ Department of Radiology, Usmanu Danfodiyo University Teaching Hospital, Sokoto, Nigeria \\ DOI: 10.29322/IJSRP.10.11.2020.p10736 \\ http://dx.doi.org/10.29322/IJSRP.10.11.2020.p10736
}

\begin{abstract}
Introduction: The term hydrocephalus literally means "water in the brain" and in this condition, excess fluid accumulates in the brain resulting in dilatation of the ventricles. It results from imbalance between cerebrospinal fluid (CSF) production and absorption. The extent of ventricular dilatation and brain compression in hydrocephalus in children is often significant and if untreated can lead to complications such as blindness and mental defiency. Therefore assessment of cerebral ventricular size is essential in the clinical management of hydrocephalus.
\end{abstract}

Aim: To evaluate hydrocephalus using linear measurements such as Evan's ratio and cerebral mantle thickness on CT images in children with hydrocephalus in sokoto, Nigeria and also compares the findings with that of other regions.

Methods: This is a four year (January 2009 - December 2013) retrospective study of 100 consecutive paediatric patients aged 0-14 years with CT diagnosis of Hydrocephalus in Radiology Department, Usmanu Danfodiyo University Teaching Hospital Sokoto. The patients CT images were retrieved from the memory of the CT scanner and the back-up recordable CDs in the CT suite of the Department. The linear measurements including maximum frontal horn distance (FHD), maximum transverse inner skull diameter (ISD) and cerebral mantle thickness were taken in axial images. The Evan's index (EI) was determined as FHD/ISD. The data was analyzed using statistical package for social sciences (SPSS) version 20.

Results: A total of 100 hydrocephalic patients who had brain CT were studied. The mean age was $3.3 \pm 3.7$ years with a range of 5 days to 14 years. The highest number of patients (64) was recorded in the age group 0-3 years. The mean EI was found to be 0.49 and the mean cerebral mantle thickness was $17.5 \mathrm{~mm}$ and $16.6 \mathrm{~mm}$ on the right and left side respectively. A statistically significant relationship was observed between EI and the type of hydrocephalus. EI was found to be higher in congenital $(p=0.015)$ and non communicating hydrocephalus $(\mathrm{p}=0.017)$.

Conclusion: The mean EI in this environment $(0.49)$ is found to be lower than the one reported in Lagos Nigeria but higher than the indices reported in other regions of the world. However, we found EI to be higher in children aged less than three years and in congenital as well as non communicating hydrocephalus as reported by previous studies in other regions.

Index Terms- Hydrocephalus, Children, Computed tomography, Linear measurements

\section{INTRODUCTION}

$\mathrm{T}$ he term hydrocephalus literally means "water in the brain" and in this condition, excess fluid accumulates in the brain resulting in dilatation of the ventricles ${ }^{1}$. It results from imbalance between cerebrospinal fluid (CSF) production and absorption. The cause of hydrocephalus is either congenital or acquired. Hydrocephalus is also classified as communicating or non communicating. In communicating hydrocephalus there is free communication between the ventricles and basal cisterns with obstruction to flow of CSF in the subarachnoid space or basal cisterns; while in non communicating hydrocephalus there is obstruction to the flow of CSF within the ventricular system ${ }^{2}$.

The extent of ventricular dilatation and brain compression in hydrocephalus in children is often significant and if untreated can lead to complications such as blindness and mental defiency ${ }^{3}$. Therefore assessment of cerebral ventricular size is essential in the clinical management of hydrocephalus. Various imaging methods play roles in the evaluation of hydrocephalus with each having its limitations. Plain radiography of the skull shows features of raised intra cranial pressure which is non specific for hydrocephalus. Ultrasonography is unsuitable in older children with fused fontanelles. Magnetic resonance imaging (MRI) has excellent soft tissue resolution but has a long time of acquisition of images which make sedation necessary in children. Computed tomography (CT) on the other hand is rapid, compatible with life support devices and often requires no sedation in children ${ }^{4}$. Computed tomography plays a central role in diagnosing hydrocephalus and also in the evaluation of treatment options, follow up and monitoring of patients 5 .

This study is intend to evaluate hydrocephalus using linear measurements such as Evan's ratio and cerebral mantle thickness on CT images in children with hydrocephalus in sokoto, Nigeria and also compares the findings with that of other regions.

\section{MATERIALS AND METHOD}

Study design: This is a four year (January 2009 December 2013) retrospective study of 100 consecutive paediatric patients aged $0-14$ years with CT diagnosis of Hydrocephalus. Cranial CT was performed at the Radiology Department Usmanu Danfodiyo University Teaching Hospital (UDUTH) Sokoto, North Western Nigeria.

Methods: All patients had brain CT scan using the spiral dual slice computed tomographic scanner of Phillip Neusoft, 
manufactured 2003. Contigous axial $5 \mathrm{~mm}$ slices were obtained pre and post intravenous contrast medium from vertex to the base of the skull. Images were reformatted in coronal and sagittal sections and stored in the memory of the CT scanner before being copied on $\mathrm{LG}$ recordable $\mathrm{CDs}$.

In this study the patients' biodata and clinical diagnosis were retrieved from the Departmental archives. The patients CT images were retrieved from the memory of the CT scanner and the back-up recordable CDs in the CT suite of the Department.

\section{Technique of CT:}

Cranial CT scans were obtained using the normal departmental protocol as follows:

After making sure there is justification for the brain CT, a brief history regarding allergy or previous reaction to contrast medium was taken. The procedure was then explained to the patient/informant. The patient then lies or was made to lie in the supine position on the CT couch. The head is placed on the head rest and supported using head immobilization pads with strap. Wedge pads and strap are used to maintain the correct head position. The couch, with the patient, was introduced into the gantry. In order to prevent unnecessary irradiation of the orbit and especially the lens, the head CT was performed with the gantry set at an angle parallel to the base of the skull. The laser beam was used for indicating the start and end points of the scan. Uncooperative patients were sedated with intravenous diazepam at a dose of $0.1 \mathrm{mg} / \mathrm{kg}$. The scout image (scanogram) was acquired from the operating console.

The scanogram was then assessed to plan axial sections of the cranium obtained from vertex to the base of the skull with slice thickness of $5 \mathrm{~mm}$, matrix size of $256 \times 256$, field of view $(\mathrm{FOV})=250 \mathrm{~mm}$, window width $=80$ and window level $=40$. The images were then inspected for presence or absent of acute intracranial haemorrhage and then intravenous contrast medium administered at a dose of $1 \mathrm{ml} / \mathrm{kg}$. Post contrast axial images were also obtained. Patients with acute intracranial haemorrhage were not given contrast medium. The obtained images were then reformatted in the sagittal and coronal planes.

The images were reported by consultant radiologist. All CT images were re-evaluated by three radiologists to reduce observer bias. The linear measurements were taken in axial images as follows:

Maximum frontal horn distance (FHD); Transverse distance between the lateral margins of the right and left frontal horns of the lateral ventricle at the level of foramen of Monro (fig 1) or at the level of maximum dilatation of the frontal horns if the foramen of Monro is not identified on the image ${ }^{6}$.

Maximum transverse inner skull diameter (ISD); The maximum diameter between inner margin of the skull measured at the same axial level where the FHD was measured ${ }^{6}$ (fig 1).

The Evans ratio was determined as the ratio of the FHD and ISD i.e FHD/ISD ${ }^{6}$.

Cerebral mantle thickness (CMT); The transverse distance between lateral margin of the anterior horn of the lateral ventricle at the point of maximum dilatation and inner margin of the skull ${ }^{6}$ (fig 2) 


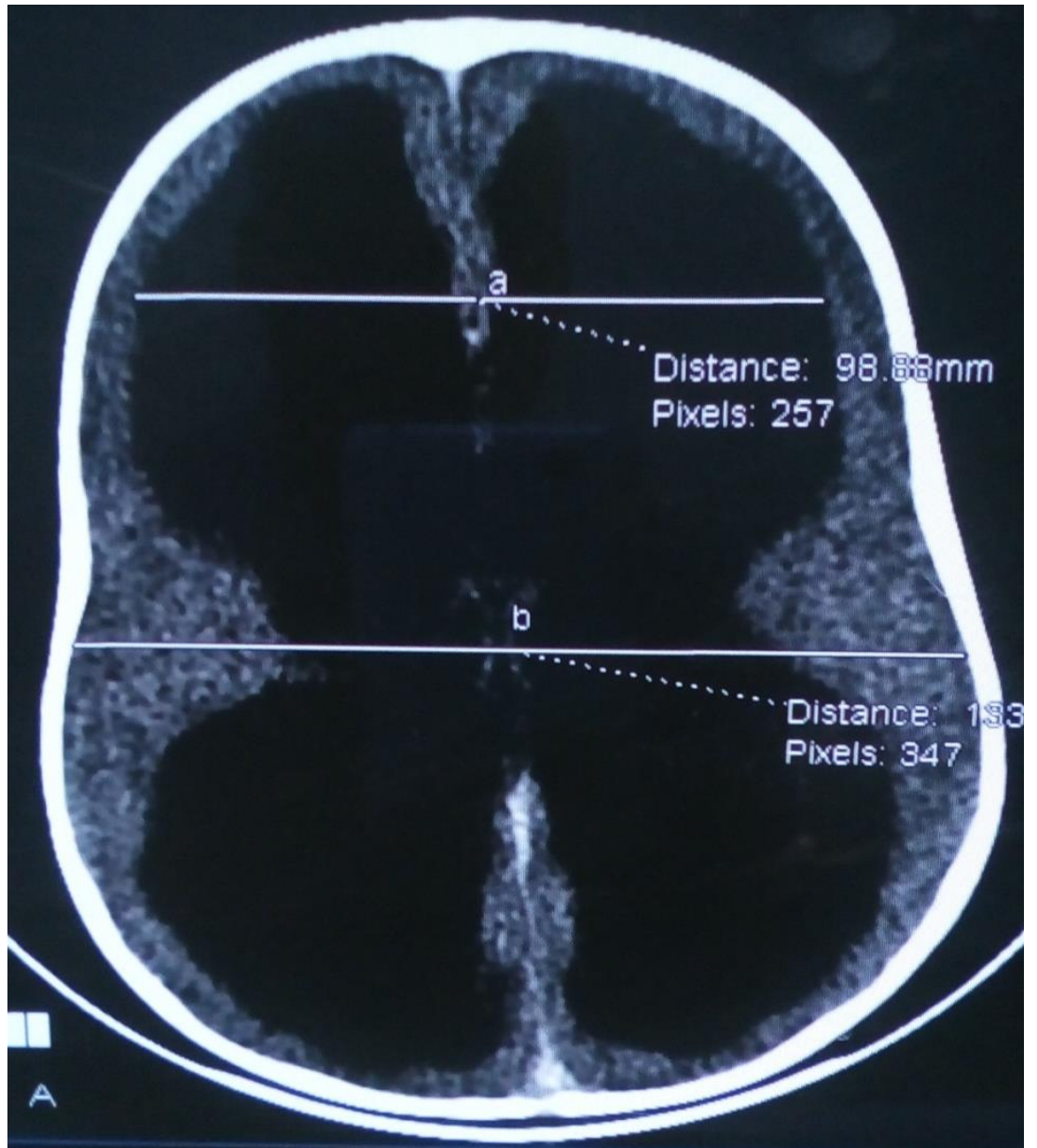

FIGURE 1: Unenhanced axial CT brain in a child with hydrocephalus at the level of the maximum frontal horns dilatation showing measurements of the maximum frontal horns distance (a) and maximum internal skull diameter (b). 


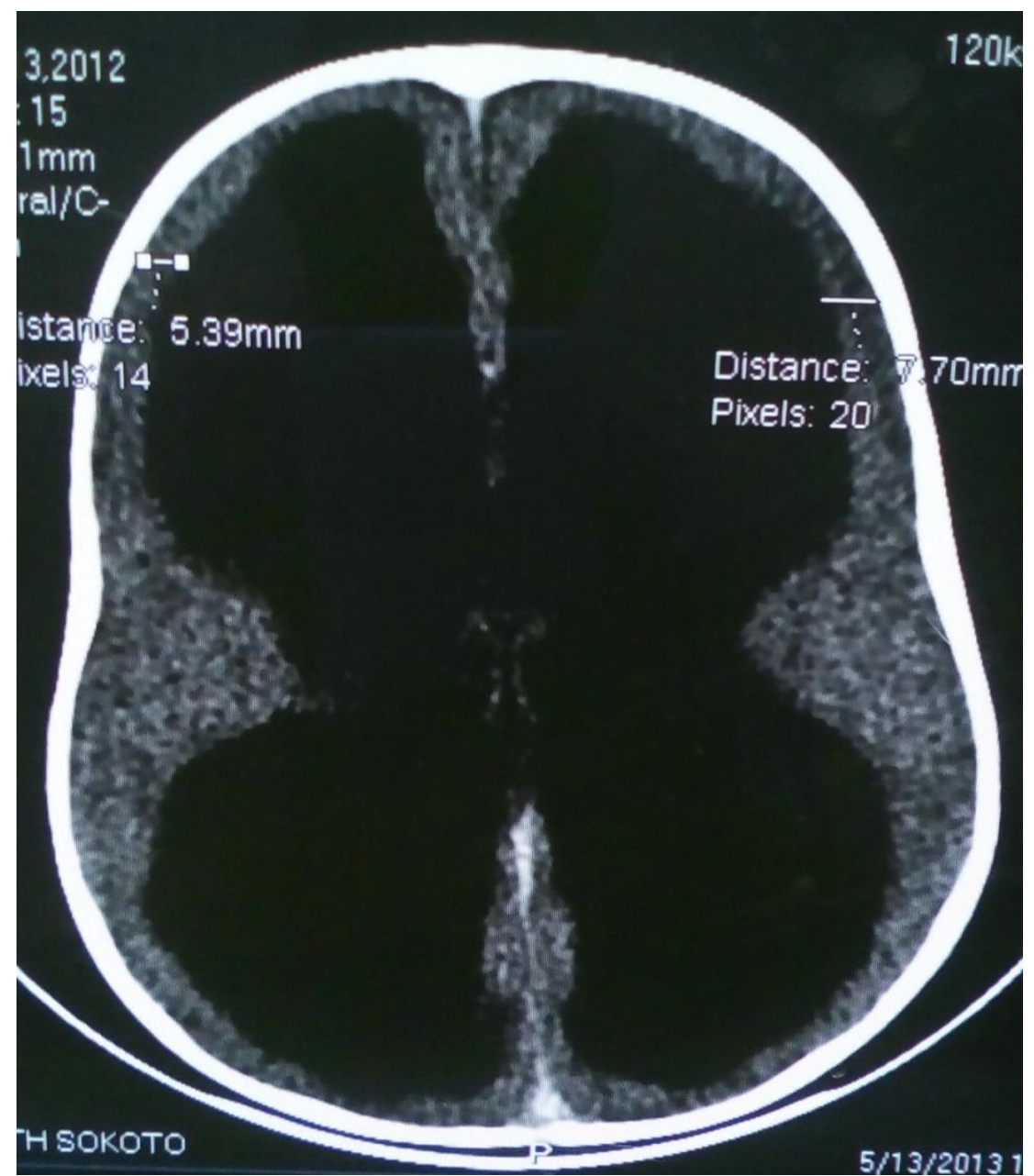

FIGURE 2: Unenhanced axial CT brain in a child with hydrocephalus at the level of the maximum dilatation of the anterior horns of the lateral ventricle showing measurements of the cerebral mantle thickness on the right and left sides.

Patients with ventriculo-peritoneal (VP) shunt tube in situ and those with brain atrophy evidenced by CT findings of dilated extraventricular CSF spaces were excluded in the study.

\section{DATA ANALYSIS}

Patients' biodata and radiological measurements were then recorded in the proforma and data was analyzed using statistical package for social sciences (SPSS) version 20. Analysis of descriptive statistics using mean and standard deviation (SD) for quantitative data (age, CMT and Evans ratio) and frequencies for qualitative data (gender and type of hydrocephalus) was done. This was followed by inferential statistics using Chi square test to determine associations between categorical variables and independent $t$ test was done for continuous variables. The result was presented in form of tables. All statistical tests were carried out using a two tail test, with level of significance set at 0.05 .

Ethical approval to conduct the study was obtained from the ethical committee of UDUTH Sokoto.

\section{RESULTS}

A total of 100 hydrocephalic patients who had brain CT were studied. Sixty two (62) of the patients were male and 38 were female with male/female ratio of 1.6:1 (table 1). The mean age was $3.3 \pm 3.7$ years with a range of 5 days to 14 years. The highest number of patients (64) was recorded in the age group 0-3 years. There was a gradual decrease in the number of patients with increasing age (table 1).

Table 1: Age distribution of patients.

\begin{tabular}{|c|c|c|c|}
\hline \multirow{2}{*}{$\begin{array}{l}\text { Age group } \\
\text { (years) }\end{array}$} & \multicolumn{2}{|c|}{ Sex of patients } & \multirow{2}{*}{ Frequency N/\% } \\
\hline & Male & Female & \\
\hline $0-3$ & 36 & 28 & 64 \\
\hline $4-6$ & 11 & 4 & 15 \\
\hline $7-9$ & 5 & 6 & 11 \\
\hline $10-12$ & 7 & 0 & 7 \\
\hline $13-14$ & 3 & 0 & 3 \\
\hline Total & 62 & 38 & 100 \\
\hline
\end{tabular}


The majority of the cases of hydrocephalus were the congenital type consisting of 54 patients with an incidence of 54\% while the acquired hydrocephalus was found in $46 \%$. Non communicating hydrocephalus was the predominant form occurring in 59 patients but the difference was not statistically significant (table 2).

Table 2: Type of hydrocephalus among study patients

\begin{tabular}{llll}
\hline Type of hydrocephalus & Frequency $(\mathbf{N})$ & $\mathbf{\%}$ & Test statistics \\
\hline Congenital & 54 & 54 & One sample binomial test \\
Acquired & 46 & 46 & $\mathrm{P}=0.194^{*}$ \\
Total & $\mathbf{1 0 0}$ & $\mathbf{1 0 0}$ & \\
& & & \\
& 41 & 41 & One sample binomial test \\
Communicating & 59 & 59 & $\mathrm{P}=0.089^{*}$ \\
Non communicating & $\mathbf{1 0 0}$ & $\mathbf{1 0 0}$ & \\
Total & &
\end{tabular}

$* \mathrm{P}>0.05$ - Not statistically significant.

The mean cerebral mantle thickness (CMT) was $17.5 \mathrm{~mm}$ \pm 10.3 on the right side and $16.6 \mathrm{~mm} \pm 9.4$ on the left (table 3 ). There was statistically significant relationship between the cerebral mantle thickness and the type of hydrocephalus. Non communicating hydrocephalus was found to be associated with less CMT implying more cerebral mantle thinning than communicating hydrocephalus $(p=0.017$ and $p=0.005$ on the right and left sides respectively) as shown in table 4 .
The mean Evan's ratio was $0.49 \pm 0.13$. The mean Evan's ratio was found to be higher in the age range 0-3 years. There was gradual decrease in Evan's ratio with age group of patients with the lowest value recorded in 12-14 age group (table 5). A statistically significant relationship was observed between Evan's ratio and the type of hydrocephalus (table 6). Evan's ratio was found to be higher in congenital $(p=0.015)$ and non communicating hydrocephalus $(\mathrm{p}=0.017)$.

Table 3: Cranial CT measurements.

\begin{tabular}{lllll}
\hline Variable (mm) & Minimum & Maximum & Mean & SD \\
\hline CMT (right) & 0.5 & 37 & 17.5 & 10.3 \\
CMT (left) & 0.9 & 35 & 16.6 & 9.40 \\
FHD & 36 & 82 & 51 & 7.4 \\
ISD & 89 & 149 & 108 & 8.3 \\
Evan's ratio & 0.31 & 0.89 & 0.49 & 0.13 \\
\hline
\end{tabular}

CMT: Cerebral mantle thickness

FHD: Frontal horn distance

ISD: Internal skull diameter

Table 4: Cerebral mantle thickness by type of hydrocephalus $* \mathrm{P}<0.05-$ Statistically significant

\begin{tabular}{lclll}
\hline Type of hydrocephalus & \multicolumn{4}{c}{ Mean cerebral mantle thickness $(\mathbf{m m})$} \\
& Right & Test statistics & Left & Test statistics \\
\hline Communicating & 20.390 & $\mathrm{t}=2.431$ & 19.195 & $\mathrm{t}=2.871$ \\
Non communicating & 15.432 & $\mathrm{df}=98$ & 13.014 & $\mathrm{df}=98$ \\
& & $\mathrm{P}=0.017^{*}$ & & $\mathrm{P}=0.005^{*}$ \\
\hline
\end{tabular}


Table 5: Cranial CT measurements by age group.

\begin{tabular}{|c|c|c|c|}
\hline \multirow{2}{*}{ Age range (yrs) } & \multicolumn{2}{|c|}{ Mean cerebral mantle thickness (mm) } & \multirow{2}{*}{$\begin{array}{l}\text { Mean Evan's ratio } \\
\text { (FHD/ISD) }\end{array}$} \\
\hline & Right & Left & \\
\hline $0-3$ & 13.3 & 11.4 & 0.76 \\
\hline $4-6$ & 15.1 & 18.3 & 0.47 \\
\hline $7-9$ & 18.1 & 18.5 & 0.43 \\
\hline $10-12$ & 18.5 & 19 & 0.43 \\
\hline $13-14$ & 22.6 & 15.8 & 0.36 \\
\hline
\end{tabular}

FHD - Frontal horn distance

ISD - Internal skull diameter

Table 6: Comparison of mean Evan's ratio between different types of hydrocephalus

\begin{tabular}{lll}
\hline Variables & Mean Evan's ratio & Test statistics \\
\hline Type of hydrocephalus & & \\
Congenital & 0.561 & $\mathrm{t}=2.321$ \\
Acquired & 0.417 & $\mathrm{df}=98$ \\
& & $\mathrm{p}=0.015 *$ \\
& & $\mathrm{t}=2.471$ \\
Communicating & 0.394 & $\mathrm{df}=98$ \\
Non communicating & 0.580 & $\mathrm{P}=0.017 *$
\end{tabular}

$* \mathrm{p}<0.05-$ Statistically significant.

\section{DISCUSSION}

Evan's ratio or index and cerebral mantle thickness gives indication of degree of ventricular dilatation in patients with hydrocephalus ${ }^{6}$. Evan's index (EI) is defined as ratio of maximum frontal horn diameter to maximum transverse diameter of the skull $^{6,7,8}$. Ventricular enlargement is when the EI is greater than or equal to $0.3 .^{8}$

In this study the mean Evan's ratio was found to be 0.49 (table 3). This value is at variance with findings of Idowu $\mathrm{et} \mathrm{al}^{6}$ in a study of non tomorous hydrocephalus in 137 children below age of six in Lagos Nigeria who reported a higher mean Evan's ratio of 0.56. Erol et $\mathrm{al}^{9}$ report a lower value of 0.45 in their study including 27 hydrocehalic children below the age of six months with pre-operative cranial CT. Radhakrishna $\mathrm{P}^{10}$ in a study titled evaluation of Evan's index by CT in hydrocephalic children also reported a lower mean EI of 0.43 . The differences in the mean Evan's ratio may be due to the inclusion of tumorous hydrocephalus and older age group of patients in this study.

A relationship between the age range of patients and Evan's ratio was observed in the index study. The highest ratio of 0.76 was found in the age range of $0-3$ years. There was gradual decrease in ratio with increasing age of patients with the lowest value of 0.36 noted in $12-14$ years age group (table 5). This trend was consistent with the findings of Radhakrishna $\mathrm{P}^{10}$ in which he reported an index of 0.47 in children below 3 years higher than in those above 3 years old (0.38). Mesiwala $\mathrm{AH}$ et a ${ }^{11}$ also reported similar findings with an index of 0.47 and 0.31 for children with congenital hydrocephalus age 1.6 years and 5 years respectively. Hydrocephalus in children may be congenital or acquired. It may also be communicating or non communicating depending on the site of the obstruction to the normal flow of the CSF. It has been reported that congenital hydrocephalus is associated with higher Evan's index than the acquired hydrocephalus ${ }^{6,12}$, In this study we also found a significantly higher Evan's ratio in the congenital ( $p$ $=0.015)$ and non communicating $(\mathrm{p}=0.017)$ types of hydrocephalus (table 6).

The mean cortical mantle thickness in this study was $17.1 \mathrm{~mm}(17.5 \mathrm{~mm}$ and $16.6 \mathrm{~mm}$ on the right and left cerebral hemisphere respectively as in table 3). However, Idowu et $a l^{6}$ in their study reported a lower mean cortical mantle thickness $(10.8 \mathrm{~mm})$. The higher value of mean cerebral mantle thickness in this study may be due to inclusion of tumorous hydrocephalus and older age range of patients studied. A statistically significant association was found between the type of hydrocephalus and cerebral mantle thickness ( $p=0.017$ and $p=0.005$ on the right and left sides respectively). The degree of cerebral mantle thinning was more in non communicating hydrocephalus than communicating hydrocephalus (table 4 ). This finding is consistent with that of Venathramana $e t a l^{13}$ in a study, evaluating the functional outcome of congenital hydrocephalus. Idowu et $a l^{6}$ also reported similar findings.

\section{CONCLUSION}

The mean Evan's index (EI) of hydrocephalic children in this environment is found to be 0.49 and the mean cortical mantle thickness is 17.5 and $16.6 \mathrm{~mm}$ on the right and left side respectively. This mean EI is lower than the one reported in Lagos Nigeria but higher than the indices reported in other regions of the world. However, we found EI to be higher in children aged less than three years and in congenital as well as non communicating hydrocephalus as reported by previous studies in other regions. 


\section{REFERENCES}

[1] Raza R, Qudsia A. Hydrocephalus in children. J Pak Med Assoc. 2005; 55(11): 502-507.

[2] Sutton D, John S, Katherine M. Intracranial lesion (1). In: Sutton D, editor. Textbook of Radiology and Imaging. 7th ed. London: Churchill Livingstone; 2003. p. 1728.

[3] Odebode TO, Shokunbi MT, Malomo AO, Familusi JB, Agbeja-Baiyeroju $\mathrm{AM}$, Ogunseyinde $\mathrm{AO}$ et al. The relationship between ventricular size and visual function in children with hydrocephalus. Afr J Med Sci. 1998; 27: 213 218. Mwangombe NJM, Omulo T. Ventriculo-peritoneal shunt infections in children at the Kenyatta National Hospital Nairobi. East Afr Med J. 2000; 77(7): 386-390.

[4] Jeff E, Gillian L. Hydrocephalus in children: Diagnostic imaging and radiol ogical characteristics 2002. www.eradiology.bidmc.harvard.edu /.../edward. (accessed online on 16th January 2013).

[5] Jon FS, Morten A, Marianne J. Childhood hydrocephalus- Is radiological morphology associated with etiology. SpringerPlus. 2013; 2(1): 1-10.

[6] Idowu O, Adebayo O. Etiology and cranial CT scan profile of non tumoral hydrocephalus in a tertiary black African Hospital. J Neurosurg Pediatr. 2011; 7: 397-400.

[7] Jamous M, Sood S, Kumar R, Ham S. Frontal and occipital horns ratio for the evaluation of small and asymmetrical ventricles. Pediatr neurosurg. 2003; 39: 17-21.

[8] Reddy VU, Hegde KV, Agrawal A, Pathapati RM, Arumulla M. Normative values for Evans index on CT scan for apparently healthy individuals. J anat Societ India. Nov 2015; 64: 137-140.

[9] Erol FS, Ozturk MB, Akgun B, Kaplan M, Cakin H, Ilhan N et al. Correlation between findings of cranial tomography and the levels of cerebrospinal fluid's nerve growth factor in hydrocephalic infants. Firat Tip Derg/Firat Med J. 2013; 18(3): 146-150.

[10] Radhakrishna P. Evalation of Evan's index by computed tomography in hydrocephalic children in tertiary hospital. Int $\mathbf{J}$ A Anat Radiol Surg. 2018;7(1): AO01-AO04

[11] Mesiwala AH, Avelino AM, Ellenbogan RG. The diagonal ventricular dimension: a method for predicting shunt malfunction on the basis of changes in ventricular size. Neurosurgery. 2002; 50(6): 1246-1252.
[12] Skiftesvik JF, Andresen M, Juhler M. Childhood hydrocephalus- is radiological morphology associated with etiology. Springer Plus. 2013; 2 : 01 10

[13] Venathramana NK, Mukundan CR. Evaluation of functional outcomes in congenital hydrocephalus. J Pediatr Neurosci. 2011; 6: 4-12.

\section{AUTHORS}

First Author - Dr. Gele IH. MBBS, FMCR Department of Radiology, Usmanu Danfodiyo University Teaching Hospital Sokoto. email; ihgele2006@yahoo.com

Second Author - Prof. Sa'idu SA. MBBS, FWACS, FMCR Department of Radiology, Usmanu Danfodiyo University Teaching Hospital Sokoto. email; sulesaidu@yahoo.com Third Author - Prof. Ma'aji SM. MBBS, FMCR Department of Radiology, Usmanu Danfodiyo University Teaching Hospital Sokoto. email; muhammadsadisu@yahoo.com

Fourth Author -Dr. Danfulani M. MBBS, FWACS Department of Radiology, Usmanu Danfodiyo University Teaching Hospital Sokoto. email; danfulo2005@gmail.com

Fifth Author -Dr. Yunusa GH. MBBS, FWACS Department of Radiology, Usmanu Danfodiyo University Teaching Hospital Sokoto. email; garusa2001@gmail.com

Sixth Author - Muhammad SB. MBBS, FWACS Department of Radiology, Usmanu Danfodiyo University Teaching Hospital Sokoto.email; muhammadsule@yahoo.com

Correspondence Author - Dr. Ibrahim Haruna Gele. MBBS, FMCR Department of radiology, Usmanu Danfodiyo University Teaching Hospital Sokoto. email; ihgele2006@yahoo.com Mobile no; +2348036066516 